SLAC-PUB-8773

Feb 2001

\title{
Study of Top-quark Production and Decay Vertices with LCD Fast Simulation [?
}

\author{
Masako Iwasaki \\ Department of Physics, University of Oregon, Eugene, OR 97403-1274, USA
}

\begin{abstract}
We report a study of top-quark reconstruction in $e^{+} e^{-} \rightarrow t \bar{t}$ events at a $500 \mathrm{GeV}$ linear collider using the LCD Fast simulator. The initial study of top-quark anomalous couplings is also reported. The final states of 4 jets and lepton as well as 6 jets are used. Using the 4 jets and lepton final state, we estimate the preliminary sensitivities for form factors at the $\gamma / Z^{0} t \bar{t}$ vertex. In the 6 jets reconstruction, we show abilities of the top-quark charge identification and the $c$-quark tagging in $\mathrm{W}$ decays.
\end{abstract}

Presented at the 5th International Linear Collider Workshop (LCWS 2000), 24-28 Oct 2000, Fermilab, Batavia, Illinois, USA

*Work supported by Department of Energy contract DE-AC03-76SF00515 (SLAC). 


\section{Introduction}

The top quark plays a special role in particle physics, due to its uniquely large mass [1]. It decays immediately, before forming a hadron. Therefore its spin information is expected to be directly transfered to its daughters. This feature provides us opportunities of probing the top couplings using the information of its daughters. The high energy future $e^{+} e^{-}$linear collider would be an ideal tool for such studies, because of its clean event environment and the possibility of initial-state polarization.

In this report, we will give a study of top-quark reconstruction in $e^{+} e^{-} \rightarrow t \bar{t}$, and the initial results of the top-quark anomalous coupling analysis. There are three kinds of final states in the $t \bar{t}$ production: (i)two $b$ jets and four jets from W's (45\%), (ii)two $b$ jets, two jets and one charged lepton(44\%), or (iii)two $b$ jets and two charged leptons(11\%). We study cases (i) and (ii), where (i) six jets and (ii) four jets and one charged lepton are in the final states. In the anomalous coupling analysis, we use the same notations of general top-quark couplings and angular definitions of polar angle, top-decay angle and W-decay angle, as Ref.[2].

\section{Event Analysis}

We generate $60,000 e^{+} e^{-} \rightarrow t \bar{t}$ events corresponding to an integrated luminosity of $80 \mathrm{fb}^{-1}$ with the PANDORA program[3], with parton showers and hadronization done by PYTHIA 6.1 [四. A top-quark mass of $175 \mathrm{GeV}$, a beam energy of $250 \mathrm{GeV}$ and $80 \%$ left-handed electron polarization are assumed. Beamstrahlung and initial state radiation are included. No other final states are generated.

To simulate the detector, we use the Linear Collider Detector (LCD) Fast simulation, assuming the Large design[5]. Charged tracks with $\mathrm{E}_{\text {track }}>200 \mathrm{MeV}$ and $|\cos \theta|<0.90$, and clusters with $\mathrm{E}_{\text {cluster }}>300 \mathrm{MeV}$ and $|\cos \theta|<0.98$ are used.

\subsection{Jets and Lepton Analysis}

In this analysis, we use only $t \bar{t} \rightarrow 4$ jets + lepton (muon or electron) events (17343 events). We apply the event-selection cuts of i) the number of charged tracks $\geq 20$, ii) there is a lepton track (muon or electron) with momentum $>20 \mathrm{GeV}$, and iii) the visible energy $>$ $300 \mathrm{GeV}$. Visible energy is calculated with charged tracks and neutral clusters. No other criteria for lepton identification are required.

Jets are reconstructed with "energy flow" objects, consisting of charged tracks and neutral clusters. Neutral clusters are selected by the absence of a track and cluster association. Here all charged tracks are extrapolated to the cluster cylindrical radius. Then the clusters which have any track with track-cluster distance $<8 \mathrm{~cm}$ are regarded as charged clusters. With this cut, we reject $93 \%$ charged while keeping $92 \%$ neutral clusters. Using the energy flow objects, except for the charged lepton, we reconstruct jets using an invariant-mass (JADE) algorithm. First we apply $Y_{\text {cut }}=0.008$ and select events which have 4 or more jets. Then 
the $Y_{\text {cut }}$ value is increased, if necessary, until the event has exactly 4 jets. The efficiency of this selection is $57 \%$ for $t \bar{t} \rightarrow 4$ jets events.

To tag the $b$ jets, we use the method developed by SLD [6]. The secondary vertex is topologically reconstructed with charged tracks, and its $P_{T^{-}}$-corrected mass $\left(M_{P_{T}}\right)$ is calculated. The jets with $M_{P_{T}}>1.8 \mathrm{GeV}$ are tagged as $b$ jets. The efficiency and purity of $61 \%$ and $95 \%$ are obtained, respectively. To identify the light-flavor ( $u d s)$ jets, we use the $N_{\text {sig }}$ method, where $N_{\text {sig }}$ is the number of tracks which have 3D impact parameter with significance $>3 \sigma$ (excluding $V^{0}$ decay tracks). We select $u d s$ jets by requiring $N_{s i g}=0$ with $87 \%$ efficiency and $77 \%$ purity.

To reconstruct $W$ 's, we search all two-jets combinations. Here we apply the flavor tagging that 1) none of two jets is tagged as a $b$ jet, and 2) at least one jet is tagged as a $u d s$ jet. Jet pairs with invariant mass within $15 \mathrm{GeV}$ of the nominal $\mathrm{W}$ mass are kept. Top-quark candidates are then formed from these W's and $b$ jets. To reduce random combinatoric background, we require $x_{E} \equiv E_{3 j e t s} / E_{\text {beam }}$ satisfies $0.9<x_{E}<1.1$. The combinations with invariant mass in the range $160 \mathrm{GeV}$ to $190 \mathrm{GeV}$ are regarded as top-quark candidates. We select 2295 (5731 for without flavor tagging) top-quark candidates, with purity of $90 \%$ $(84 \%)$. In this sample, $89 \%(56 \%)$ of the candidates have the correct $b$ and $\mathrm{W}$ assignment. The mass resolution for the reconstructed top quark is $7.8 \mathrm{GeV}(9.2 \mathrm{GeV})$.

Then we reconstruct the polar angle $(\theta)$ of top quark, top-decay angle $\left(\chi_{t}\right)$ and $\mathrm{W}$-decay angle $(\chi)$. The charge of the top quark is determined by the charge of the lepton. In the 4 jets and lepton analysis, we only reconstruct one $t(\bar{t})$ quark, which has 3 jets in the final state. Since the final state of the other $\bar{t}(t)$ quark includes a neutrino which can not be detected, we assume that the $\bar{t}(t)$ quark has the same energy and the opposite momentum as the reconstructed $t(\bar{t})$ quark. We also assume that 1) $b$ jet and $W$ are back-to-back in the top rest frame, and 2) lepton and neutrino are back-to-back in the $\mathrm{W}$ rest frame, to reconstruct the top-decay and W-decay angles. Fig. 1 shows the reconstructed angular distributions. Reconstruction efficiencies in $\cos \chi_{t}>0.6$ and $\cos \chi<0$ are significantly dropped because of the acceptance cut. The angular resolutions are $35 \mathrm{mrad}, 69 \mathrm{mrad}$ and $115 \mathrm{mrad}$ for $\theta, \chi_{t}$ and $\chi$, respectively.

Using the angular distributions, we estimate the preliminary sensitivities of 0.023 and 0.034 (normalized) for $F_{1 A}^{\gamma}$ and $F_{1 A}^{Z}$, respectively, at the $\gamma / Z^{0} t \bar{t}$ vertex for an integrated luminosity of $80 \mathrm{fb}^{-1}$ with $80 \%$ left-handed electron beams.

\subsection{Jets Analysis}

In this study, we use $27730 t \bar{t} \rightarrow 6$ jets events in the generated $t \bar{t}$ sample described above. For the event selection, we require i) the number of charged tracks $\geq 30$ and ii) visible energy $>350 \mathrm{GeV}$. The energy flow objects are used to form jets. As before, we first apply $Y_{\text {cut }}=0.003$ to select events with 6 or more jets, then increase $Y_{\text {cut }}$ until the event has exactly 6 jets. We require that an event has exactly $2 b$ jets, where $b$ jets are identified by a cut $M_{P_{T}}$ $>1.8 \mathrm{GeV}$. After identifying the two $b$ jets, we exclusively reconstructed two W's from the remaining 4 jets. Top-quark candidates are then formed from W's and $b$ jets. We require at 

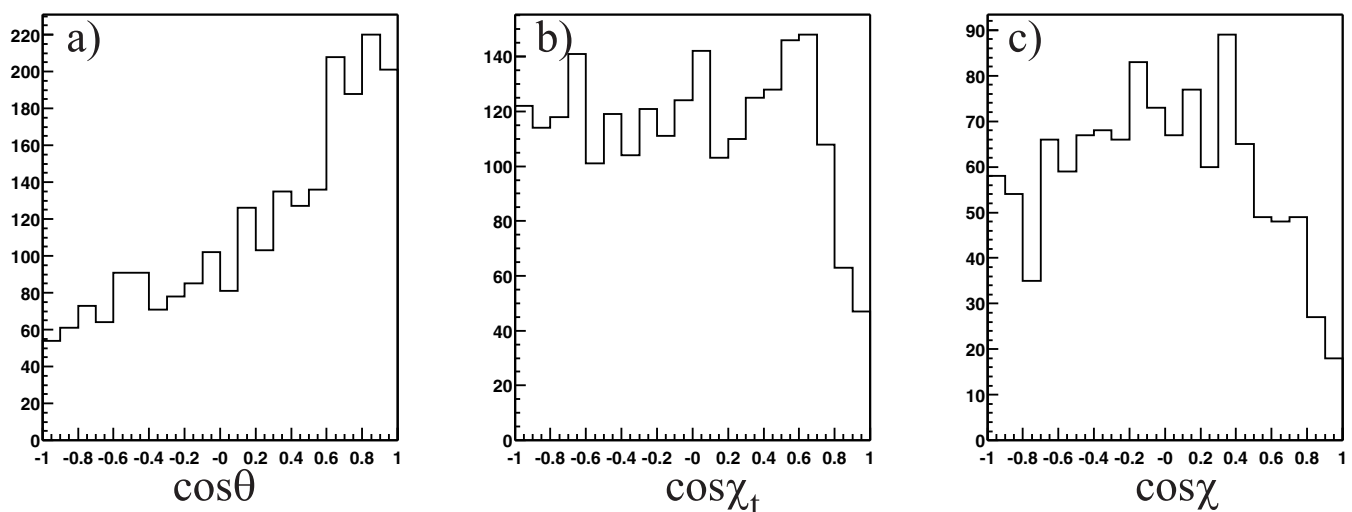

Figure 1: The angular distributions of a) $\cos \theta, \mathrm{b}) \cos \chi_{t}$ and c) $\cos \chi$ for the reconstructed $t \bar{t}$ candidates. The definitions of the $\chi_{t}$ and $\chi$ are in the text.

least one $\mathrm{W}$ candidate has an invariant mass within $15 \mathrm{GeV}$ of the nominal $\mathrm{W}$ mass, and at least one top candidate satisfies $160 \mathrm{GeV}<M_{\text {top }}<190 \mathrm{GeV}$.

To identify the charge of the top quarks, we measure Vertex Charge of $b$ jets. Here the Vertex Charge is a charge sum of the secondary vertex reconstructed by the topological vertexing. We require at least one of two $b$ jets has the non-zero Vertex-Charge value. We discard the events which have the same-sign $b$ jets. The efficiency of this cut is $57 \%$, and the charge of the top quark is determined with $83 \%$ purity. The provability of the correct assignment of $b$ and $\mathrm{W}$ in the top-quark candidates is $78 \%$. We reconstruct the top-quark polar angle $\theta$ and the top-decay angle $\chi_{t}$ with resolutions of $\Delta \theta=48 \mathrm{mrad}$ and $\Delta \chi_{t}=88$ mrad.

To reconstruct the $\mathrm{W}$-decay angle, we tag the $c$ quark in the $\mathrm{W}$ decay using the $P_{T^{-}}$ corrected mass $\left(M_{P_{T}}\right)$ and momentum of the secondary vertex $\left(P_{V T X}\right)$. We identify the $c$ jets with the criteria i) $20 \times M_{P_{T}}-P_{V T X}<10$ ii) $0.6 \mathrm{GeV}<M_{P_{T}}<1.8 \mathrm{GeV}$, and iii) $P_{V T X}>10 \mathrm{GeV}$. The purity and efficiency for $c$ quarks are $98 \%$ and $33 \%$, respectively. Here we get the high purity because there is little $b$ background in the reconstructed W's. We select $689 c$-jet candidates, and reconstruct the $\mathrm{W}$-decay angles with the angular resolution of $90 \mathrm{mrad}$.

Since we use the heavy-flavor tagging and the charge identification with the Vertex Charge in this analysis, the Vertex Detector performance is expected to be important. Changing the inner radius of the Vertex Detector from $1 \mathrm{~cm}$ to $2 \mathrm{~cm}$, we lose $1 \%$ of the top-quark reconstruction efficiency and $13 \%$ of the c-quark tagging efficiency in $\mathrm{W}$ decays. Therefore the Vertex Detector performance is important especially for the $c$-quark tagging. 


\section{Conclusion}

We have studied the top-quark reconstruction in $e^{+} e^{-} \rightarrow t \bar{t}$ events at a $500 \mathrm{GeV}$ linear collider using the LCD Fast simulator.

In the 4 jets and lepton analysis, we estimate the preliminary sensitivities of $F_{1 A}^{\gamma}$ and $F_{1 A}^{Z}$ at the $\gamma / Z^{0} t \bar{t}$ vertex for an integrated luminosity of $80 \mathrm{fb}^{-1}$ with $80 \%$ left-handed electron beams. To estimate the other coupling sensitivities, the detailed acceptance correction and background studies are necessary.

In the 6 jets analysis, we determine the charge of the top quark using the Vertex Charge of $b$ jet with $83 \%$ purity. We also apply the $c$-quark tagging in $\mathrm{W}$ decays. The Vertex Detector performance is important especially for the $c$-quark tagging.

\section{References}

[1] CDF collaboration, T. Affolder et al., Phys. Rev. D63 032003 (2001);

D0 collaboration, B. Abbott et al., Phys. Rev. D58 052001 (1998).

[2] C. Schmidt, Phys. Rev. D54 3250 (1996).

[3] M. Peskin, hep-ph/9910519 (1999).

[4] T. Sjöstrand, Comp. Phys Comm. 8274 (1994).

[5] M. Iwasaki and T. Abe, "LCD ROOT Simulation and Analysis Tools", These proceedings.

[6] D. Jackson, Nucl. Inst. Meth. A388 247 (1997). 